\title{
Short-term Forecast of Multiple Loads in Integrated Energy System Based on IPSO-WNN
}

\author{
Cao Yuwei ${ }^{1, *}$, Zeng Ming ${ }^{1}$, Jiang Shigong ${ }^{2}$, Yang Weihong ${ }^{2}$, Shi Pengjia ${ }^{3}$, and Guo Xiaopeng ${ }^{1}$ \\ ${ }^{1}$ North China Electric Power University, 102206, Beijing China \\ ${ }^{2}$ State Grid Economic and Technological Research Institute Co., Ltd., 102209, Beijing China \\ ${ }^{3}$ State Grid Fujian Electric Power Co. Ltd., 350012, Fuzhou China
}

\begin{abstract}
Accurate short-term energy load forecasting has a considerable influence on the economic scheduling and optimal operation of integrated energy system. This study proposes an improved particle swarm optimization-wavelet neural network (IPSO-WNN) method for short-term load forecasting of integrated energy system. First, Kendall rank correlation coefficient in Copula theory is used to analyze the correlation among the influencing factors, through which the influencing factors with strong correlation are selected as input variables of the model. Secondly, chaos algorithm and adaptive weight selection strategy are introduced in the POS-WNN forecasting model to improve the prediction accuracy. Therefore, a shortterm load forecasting model of integrated energy system based on IPSO-WNN is established. Finally, the analysis of examples shows that the load prediction accuracy is significantly improved based on the IPSOWNN model compared with the traditional forecasting model.
\end{abstract}

\section{Introduction}

The integrated energy system (IES) is a comprehensive energy supply platform, including plenty of energy sources such as cold, heat, and electricity ${ }^{[1]}$. The shortterm load forecast of the IES is the premise of its operation and scheduling ${ }^{[2]}$.

Scholars have used a variety of advanced methods for load forecasting. Reference [3] introduced chaos disturbance factor in cuckoo algorithm and established the Elman-IOC short-term electric load forecasting model. Reference [4] proposed a short-term load forecasting method based on deep belief network applied in a complex environment. Reference [5] proposed a new method for short-term load forecasting sensitive to external factors based on empirical mode decomposition and feature correlation analysis. Although the above studies have high forecasting accuracy, they are only used for electric load forecasting and do not consider multiple loads such as electric, gas, heat, and cold loads. Reference [6] used the ARMAX model to make multiple load forecasting. Reference [7] proposed a short-term combined forecasting method of multiple loads based on deep structure multi-task learning. Reference [8] quantitatively analyzed the correlation between cold and heat loads based on Copula theory, which improved the load prediction accuracy of the IES. The above load forecasting studies have considered the coupling relationship of multiple loads, but few studies have considered all the multiple loads including electric, gas, heat, and cold loads. The exploration of the coupling relationship between multiple loads is still in progress, which needs to be further studied.

The multiple loads in the IES are not only related to their own historical data but also affect each others. Therefore, this paper uses an improved wavelet neural network (WNN) to make short-term forecasting for electric, heat, cold and gas loads. Firstly, based on Copula theory, the correlation between loads and temperature factors is analyzed to select the appropriate influencing factors. Secondly, chaos algorithm is added to particle swarm optimization (PSO) to optimize the particle inertia weight. The improved PSO is used to optimize the connection weights and wavelet function parameters in the WNN to construct an IPSO-WNN multiple load forecasting model. Finally, an example is used to prove the prediction accuracy of the method.

\section{Correlation analysis based on Copula theory}

In order to more intuitively show the correlation between multiple loads in the IES and the relationship between them and weather factors, this paper uses Copula theory for correlation analysis, which describes the non-linear correlation between variables.

Sklar theorem effectively combines multivariate distribution with Copula function ${ }^{[8]}$. If the edge distribution function $F_{1}\left(a_{1}\right), F_{2}\left(a_{2}\right), \cdots, F_{n}\left(a_{n}\right)$ of the joint distribution function $F(A)$ for the random variable $A=\left[A_{1}, A_{2}, \cdots, A_{n}\right]^{T}$ are continuous, then there is a Copula function $\mathrm{C}$ that satisfies

\footnotetext{
* Corresponding author: $1204301572 @$ qq.com
} 


$$
F(X)=C\left(F_{1}\left(a_{1}\right), F_{2}\left(a_{2}\right), \cdots, F_{n}\left(a_{n}\right)\right)
$$

Spearman rank correlation coefficient and Kendall rank correlation coefficient are commonly used for quantitative analysis of the nonlinear correlation between random variables. Kendall rank correlation coefficient not only describes the nonlinear relationship between variables, but also has all Copula functions. Therefore, this paper uses Kendall rank correlation coefficient $\tau$ to describe the relationship between various variables ${ }^{[9]}$. If the edge distribution functions $F_{1}(j)$ and $F_{2}(k)$ of the joint distribution function $F(j, k)$ of the two-dimensional random variable $(J, K)$ exist and are continuous, they are represented by $u$ and $v$, respectively. After calculation and goodness-of-fit test, the Copula function obtained is represented by $C(u, v)$, then the Kendall rank correlation coefficient $\tau$ can be expressed as

$$
\tau=4 \int_{0}^{1} \int_{0}^{1} C(u, v) \mathrm{d} C(u, v)-1
$$

When $\tau>0$, the variables are positively correlated; when $\tau<0$, the variables are negatively correlated; when $\tau=0$, the correlation cannot be determined.

In general, all load distributions follow Gaussian distribution. Therefore, this paper chooses the Gaussian Copula function to couple the multiple loads, and the calculation is shown in (3):

$$
\begin{aligned}
C_{n}(u, v)= & \frac{1}{\sqrt{\left(1-\theta_{n}^{2}\right)}} \\
& \exp \left\{\frac{-\left[\left(\phi_{n}^{-1}(u)\right)^{2}+\left(\phi_{n}^{-1}(v)\right)^{2}-2 \theta_{n} \phi_{n}^{-1}(u) \phi_{n}^{-1}(v)\right]}{2\left(1-\theta_{n}^{2}\right)}\right\} \\
& \exp \left\{-\frac{\left(\phi_{n}^{-1}(u)\right)^{2}\left(\phi_{n}^{-1}(v)\right)^{2}}{2}\right\}
\end{aligned}
$$

Where, $\phi_{n}^{-1}$ is the inverse function of the standard normal distribution.

The Kendall rank correlation coefficient corresponding to the Gaussian Copula function can be expressed as:

$$
\tau=\frac{2}{\pi} \arcsin \theta
$$

From equations (2)-(4), the Kendall rank correlation coefficient and Copula function value between multiple loads can be calculated.

In this section, the Kendall rank correlation coefficients are used to select the strongly correlated variables as the input variables of the load forecasting model, and the Copula function values of the strongly correlated variables are entered as new data, which provides a basis for forecasting various loads.

\section{Improved PSO-WNN prediction method}

\subsection{Wavelet neural network model}

The coupling of multiple loads in the IES is reflected in all links. Therefore, this section introduces a neural network model to express the complex relationship between various loads.

WNN uses the wavelet function as the activation function in the hidden layer. It uses the multi-scale resolution and good time-frequency analysis capabilities of the wavelet function combined with the self-learning ability of the neural network (see Fig.1) ${ }^{[10]}$. From the input layer to the hidden layer, this paper uses Morlet wavelet function and its corresponding translation amount and scaling amount to calculate, as shown in (5)(7). The connection weight is used to calculate from the hidden layer to the output layer, as shown in (8).

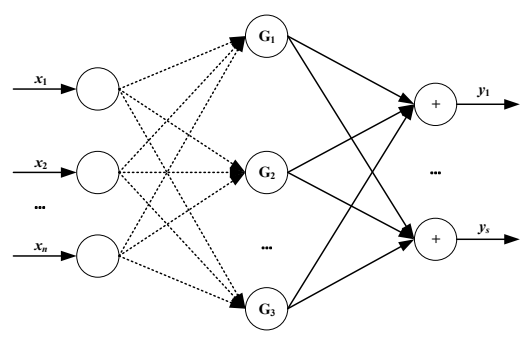

Fig.1. Structure diagram of wavelet neural network.

$$
\begin{gathered}
G_{m}\left(x_{1}, x_{2}, \cdots, x_{n}\right)=\prod_{i=1}^{n} \psi_{a_{m}, b_{m}}\left(x_{i}\right) \forall m=1,2, \cdots, R \\
\psi_{a_{m}, b_{m}}\left(x_{i}\right)=\psi\left(\frac{x_{i}-b_{m}}{a_{m}}\right) \\
\psi(x)=e^{-0.5 x^{2}} \cos (5 x) \\
y_{s}=\sum_{m=1}^{R} w_{m}^{s} G_{m}\left(x_{1}, x_{2}, \cdots, x_{n}\right)
\end{gathered}
$$

Where, $G_{m}$ is the output of the hidden layer $m ; \psi(x)$ is the Morlet wavelet function in the wavelet function; $a_{m}$ and $b_{m}$ are the amount of translation and scaling of the wavelet function, respectively; $x$ is the input amount, $y$ is the output amount; $w_{m}^{s}(\mathrm{~s}=1,2,3)$ is the connection weight.

\subsection{Particle swarm optimization}

Traditional WNN uses Back Propagation algorithm to optimize parameters, which may lead to sparse information transmitted by the neural network, causing poor load forecasting performance of the IES. PSO has fast optimization speed and good optimization effect ${ }^{[11]}$. It can optimize connection weights and wavelet parameters in WNN. The position and velocity update formulas are shown in (9) and (10):

$$
\begin{aligned}
V_{t}^{c+1}= & w \cdot V_{t}^{c}+k_{1} \operatorname{rand}_{1} \cdot\left(P_{b e s t}-X_{t}\right) \\
& +k_{2} \operatorname{rand}_{2} \cdot\left(H_{\text {best }}-X_{t}\right)
\end{aligned}
$$




$$
X_{t}^{c+1}=X_{t}^{c}+V_{t}^{c+1}
$$

Where, $V_{t}^{c}$ and $X_{t}^{c}$ are the velocity and position of particle $t$ at $c$ iteration; $w$ is the particle weight coefficient; rand $_{1}$ and rand $_{2}$ are randomly generated in $[-1,1] ; k_{1}$ and $k_{2}$ are learning factors; $H_{\text {best }}$ and $P_{\text {best }}$ are the global and individual optimum, respectively.

The convergence speed and optimization effect of PSO are affected by the weight coefficient, so it is crucial to select a suitable weight coefficient $w$. Different weight strategies are established due to the different weight requirements of particles. $P_{g}$ is the fitness of the current global optimal particle. $\bar{\zeta}$ is the current average fitness value of the particle. $\zeta_{p}$ is the average fitness value superior to the particle. $\zeta_{b}$ is the fitness of particle $b$.

When $\zeta_{b}>\bar{\zeta}$, particle $b$ is the farthest from the optimal position, so the inertia weight coefficient is relatively large. The calculation of its inertia weight is shown in (11) and (12):

$$
\begin{gathered}
w=w_{\text {avg }}+\frac{w_{\max }-w_{\min }}{2} \cdot \text { rand } \\
w_{\text {avg }}=\frac{w_{\max }-w_{\min }}{2}
\end{gathered}
$$

Where, $w_{\max }$ and $w_{\min }$ are the maximum and minimum weights respectively; $w_{\text {avg }}$ is the average weight; rand is a random value in $[0,1]$.

When $\zeta_{b}<\zeta_{p}$, particle $b$ is closer to the optimal position, so the inertia weight coefficient should be smaller. The calculation of inertia weight is shown in equation (13):

$$
w=w_{a v g}-\left|\frac{\zeta_{t}-\zeta_{p}}{P_{g}-\zeta_{p}}\right| \cdot\left(w_{a v g}-w_{\min }\right)
$$

When $\zeta_{p}<\zeta_{b}<\bar{\zeta}$, the calculation of inertia weight should use the non-linear decreasing weight method, as shown in equation (14):

$$
w=w_{\max }-\frac{\left(w_{\max }-w_{\min }\right) \cdot \text { iter }}{\text { maxgen }}
$$

Where, iter is the current number of iterations; maxgen is the total number of iterations.

\subsection{Chaos algorithm}

To solve the problem that PSO is easily lost in the local optimal solution, a chaos algorithm is introduced in PSO iterative process ${ }^{[12]}$. To improve forecasting speed, the chaos search is only performed on the current optimal particle after each iteration. If the new particle produced after search is better than the optimal particle, the optimal substitution is performed. The process is shown in $(15)$ - (17).

$P_{\text {best }}=\left(p_{1}, p_{2}, \cdots, p_{D}\right)$ is the individual optimal position, and each bit $p_{t}$ in $P_{\text {best }}$ is mapped to the chaotic variable $\gamma_{d}^{c}, \gamma_{d}^{c} \in[0,1]$, as shown in (15).

$$
\gamma_{d}^{c}=\frac{p_{d}^{c}-p_{\max , d}}{p_{\max , d}-p_{\min , d}}
$$

Where, $d=(1,2, \cdots, D) ; P_{\min , d}$ and $P_{\text {max }, d}$ are the lower and upper limits of the search space of the $d$-th dimensional variable, respectively. Logistic iterative equation is used to process $\gamma_{d}^{c}$, as shown in (16):

$$
\gamma_{d}^{c+1}=\eta \gamma_{d}^{c}\left(1-\gamma_{d}^{c}\right)
$$

Where, $\eta$ is the chaos coefficient. The inverse chaotic variable $\gamma_{d}^{c}$ after iteration is mapped to the original space solution as shown in (17):

$$
x_{d}^{c}=\left(p_{\max , d}-p_{\min , d}\right) \cdot \gamma_{t}^{c}+p_{\min , d}
$$

A new solution $x_{d}^{c}$ can be obtained. If the particle's fitness is better than $P_{b e s t}$, the position replacement is needed.

\subsection{Improved PSO-WNN prediction algorithm}

In this paper, chaos algorithm is introduced into PSO and particle inertia weight is selected based on particle fitness. IPSO is used to optimize the connection weights and the translation and scaling of wavelet function. A WNN multiple loads forecasting model is constructed to perform short-term load forecasting on the IES. The prediction algorithm process is shown in Fig. 2.

(1) Input the historical load data of cold, heat, gas and electric, as well as the temperature, and daily type data, and perform normalization.

(2) Initialize connection weight and the amount of translation and expansion in the wavelet function. The initial individual and global optimum are obtained through particle swarm initialization.

(3) Update the speed and position of the particles. Calculate the fitness of each particle and update the individual and global optimums. Calculate weight $w$ based on equations (11) - (14).

(4) Chaos search is performed on the current optimal particles based on equations (15) - (17). If the new particle is better than the current one, replace it.

(5) Continue if the conditions for the next step are met. If not, return to step (3).

(6) Obtain the optimal initial connection weights and wavelet parameters. Construct WNN prediction model, predict multiple loads and output the prediction results. 


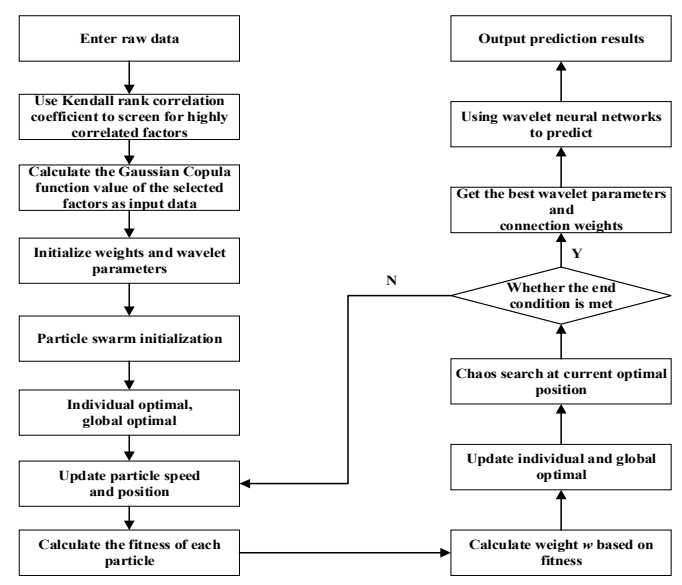

Fig.2. Load forecasting flow chart.

\section{Example analysis}

This study uses an IES in Guangzhou to verify the proposed method. The IES includes CCHP, photovoltaic plant, energy storage, heat pump and chiller units, which provide users with the load of cold, heat, electric and gas demands. The system's data for the first twenty days in November 2019 are selected as historical data, and the data of the last ten days are used as training data to predict the cold, heat, electric and gas loads of the first day in December 2019.

\subsection{Correlation analysis}

Based on historical data, the Kendall rank correlation coefficients are calculated according to Section 2, and the highly relevant variables are selected as input variables of the load forecasting model to provide a basis for future load forecasting. The calculation results are shown in Table 1.

As can be seen from Table 1, there is no significant difference between the correlation between electric load and thermal load and that between electric load and gas load, and both are positively correlated. However, the correlation coefficients are relatively small. On the contrary, the correlation coefficient between electric load and cold load and the correlation coefficient between electric load and temperature are larger. There is a significantly positive correlation between electric load and cold load, while a significantly negative correlation between heat load and temperature. Therefore, the load forecasting of the IES should take into account the coupling effects between electric and cold loads, electric load and temperature, heat load and temperature, and cold load and temperature.

Table 1. Kendall rank correlation coefficient.

\begin{tabular}{|c|c|c|c|c|c|}
\hline & Electric & Heat & Cold & Gas & Temperature \\
\hline Electric & 1 & 0.177 & 0.431 & 0.187 & 0.451 \\
\hline Heat & 0.177 & 1 & 0.284 & 0.043 & -0.473 \\
\hline Cold & 0.431 & 0.284 & 1 & 0.081 & 0.371 \\
\hline Gas & 0.187 & 0.043 & 0.081 & 1 & -0.122 \\
\hline Temperature & 0.451 & 0.473 & 0.371 & 0.122 & 1 \\
\hline
\end{tabular}

\subsection{Result analysis}

In order to verify the effectiveness of the proposed prediction method, three scenarios are set up:

Scenario 1: Considering the coupling of cold, heat, electric, and gas loads, an IPSO-WNN forecasting model is used.

Scenario 2: Without considering the coupling of cold, heat, electric, and gas loads, a IPSO-WNN forecasting model is used.

Scenario 3: Considering the coupling of cold, heat, electric, and gas loads, a PSO-WNN forecasting model is used.

The prediction results of cold load, electric load, gas load, and heat load in the three scenarios are shown in Fig. 3 - Fig. 6.

In this study, mean absolute percent error (MAPE) and weighted mean accuracy (WMA) are selected as the indicators to evaluate the accuracy of the model. The calculation formulas are shown in (18) - (20). The prediction accuracy is shown in Table 2.

$$
\begin{gathered}
M A P E=\frac{1}{N} \sum_{t=1}^{N}\left|\frac{y_{t}-y_{t}^{\prime}}{y_{t}}\right| \times 100 \% \\
M^{\prime}=1-M A P E \\
W M A=\sigma_{e} M_{e}^{\prime}+\sigma_{h} M_{h}^{\prime}+\sigma_{c} M_{c}^{\prime}
\end{gathered}
$$

Where, $y_{t}$ is the actual value; $y_{t}^{\prime}$ is the predicted value; $\sigma_{e}, \sigma_{h}$ and $\sigma_{c}$ are the weights of electricity, heat and cold load, respectively. The weight coefficients in this study are set to $0.6,0.2$ and 0.2 , respectively.

Table 2. Prediction accuracy and prediction time of three kinds of scenarios.

\begin{tabular}{|c|c|c|}
\hline & $\begin{array}{c}\text { Prediction accuracy } \\
(\boldsymbol{W M A} / \mathbf{\%})\end{array}$ & $\begin{array}{c}\text { Prediction time } \\
(\boldsymbol{t} / \mathbf{s})\end{array}$ \\
\hline Scenario 1 & 97.88 & 313 \\
\hline Scenario 2 & 96.12 & 277 \\
\hline Scenario 3 & 95.77 & 289 \\
\hline
\end{tabular}

It can be seen from Table 2 that the accuracy of scenario 2 is higher than that of scenario 1 . Therefore, the prediction accuracy can be improved when the coupling among electric, gas, heat and cold load is considered. In addition, the prediction error values of scenario 1 are all smaller than those of scenario 3 , and the differences are large, indicating that the prediction accuracy of the IPSO-WNN model is significantly better than that of the PSO-WNN model. This means that the IPSO-WNN model has a strong tracking ability for various loads, which can overcome the shortcomings of the traditional algorithm such as poor convergence and easy falling into local optimal, and can effectively improve the prediction accuracy.

In addition, in the three scenarios, the prediction speed of scenario 2 is the fastest because the coupling relationship between various loads is not considered, resulting in fewer input variables. The introduction of chaos algorithm increases the complexity of particle 
swarm optimization and reduces the convergence speed of IPOS-WNN, resulting in that the prediction time of scenario 1 is longer than that of scenario 3 .

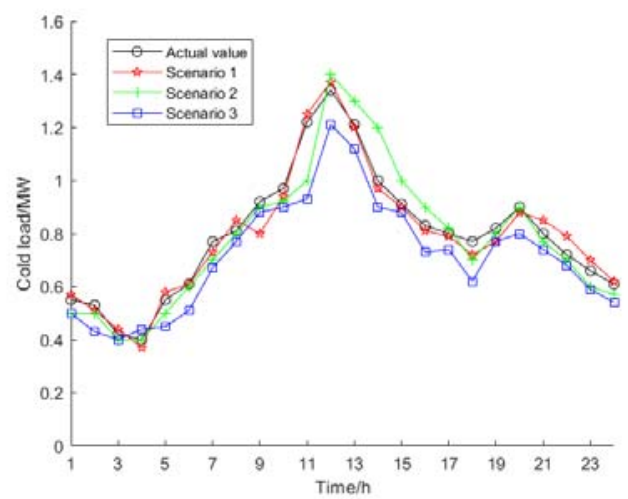

Fig.3. Cold load prediction results.

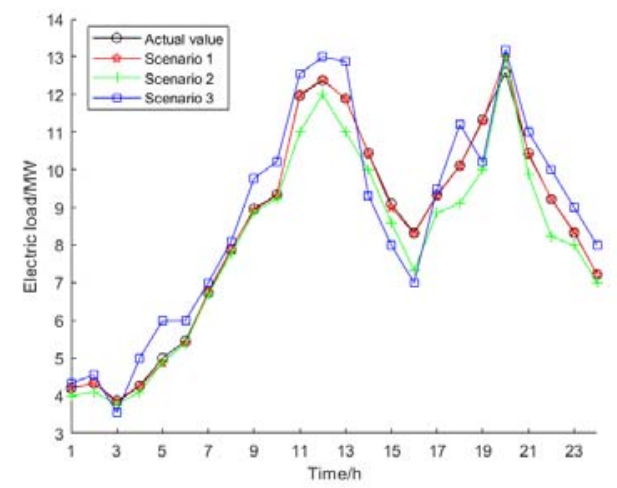

Fig.4. Electric load prediction results.

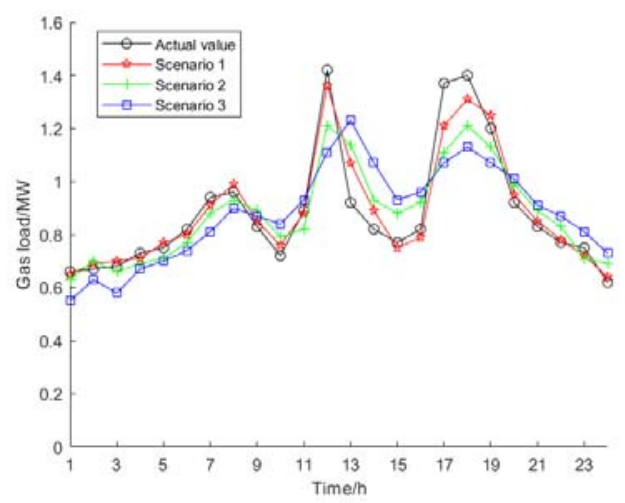

Fig.5. Gas load prediction results.

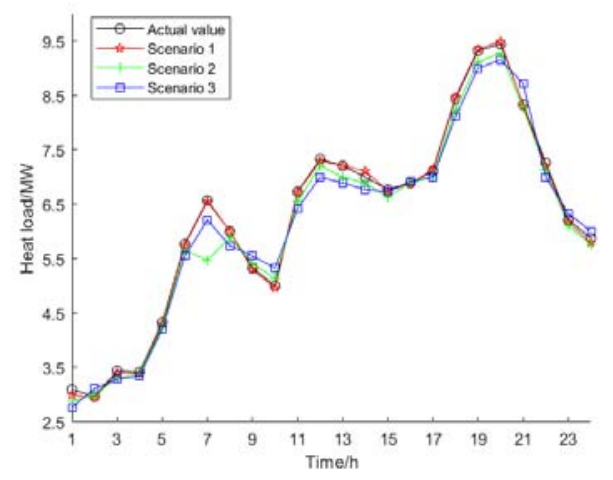

Fig.6. Heat load prediction results.

\section{Conclusion}

In order to improve the load prediction accuracy of IES, a short-term load forecasting method based on IPSOWNN is proposed. The Kendall rank correlation coefficient is used to select appropriate factors as input variables of the model. Chaos algorithm and adaptive weight selection strategy are introduced in the POSWNN prediction model to improve the prediction accuracy of the model. Through example analysis, the IPSO-WNN load forecasting method of IES is verified to effectively improve the prediction accuracy, speed and the performance of forecasting models.

\section{Acknowledgement}

This work was supported by State Grid Science and Technology Project "Research on collaborative planning technology for the fusion development of intelligent distribution network and ubiquitous power internet of things" (5400-201956447A-0-0-00).

\section{References}

1. J. Wu, J. Yan, H. Jia, N.D. Hatziargyriou, N. Djilali, H. Sun, Appl. Energ. 167, 155 (2016)

2. B. Zhao, Z. Wang, W. Ji, X. Gao, X. Li, Power Syst. Technol. 43, 4370 (2019)

3. F. Yang, Y. Wang, L. Wang, Q. Chang, Electrical Measurement \& Instrumentation 56, 32 (2019)

4. X. Kong, F. Zheng, Z. E, J. Cao, X. Wang, Automat. Elecrton. Power Syst. 42, 133 (2018)

5. X. Kong, C. Li, F. Zheng, L. Yu, X. Ma, Automat. Elecrton. Power Syst. 43, 46 (2019)

6. M. Liu, Y. Shi, F. Fang, IEEE Trans. Control Syst. Technol. 23, 1672 (2015)

7. J. Shi, T. Tan, J. Guo, Y. Liu, J. Zhang, Power Syst. Technol. 42, 698 (2018)

8. J. Ma, W. Li, S. An, Z. Zhang, Journal of Qingdao University(Engineering \& Technology Edition) 33, 56 (2018)

9. W. Lu, G. Li, C. Dong, R. Quan, Distributed Energy 4, 1 (2019)

10. Y. Gao, S. Li, F. Chi, L. Ge, D. Zhang, Proceedings of the CSU-EPSA 31, 62 (2019)

11. H. Cheng, Z. Huang, Electronic Measurement Technology 42, 94 (2019)

12. J. Tang, H. Ma, Journal of Yunnan University(Natural Sciences Edition) 41, 1123 (2019) 